\title{
On maximal actions and $w$-maximal actions of finite hypergroups
}

\author{
Bangteng Xu
}

Received: 11 September 2006 / Accepted: 30 May 2007 /

Published online: 13 June 2007

(C) Springer Science+Business Media, LLC 2007

\begin{abstract}
Sunder and Wildberger (J. Algebr. Comb. 18, 135-151, 2003) introduced the notion of actions of finite hypergroups, and studied maximal irreducible actions and $*$-actions. One of the main results of Sunder and Wildberger states that if a finite hypergroup $K$ admits an irreducible action which is both a maximal action and a *action, then $K$ arises from an association scheme. In this paper we will first show that an irreducible maximal action must be a $*$-action, and hence improve Sunder and Wildberger's result (Theorem 2.9). Another important type of actions is the so-called $w$-maximal actions. For a $w$-maximal action $\pi: K \rightarrow \operatorname{Aff}(X)$, we will prove that $\pi$ is faithful and $|X| \geq|K|$, and $|K|$ is the best possible lower bound of $|X|$. We will also discuss the strong connectivity of the digraphs induced by a $w$-maximal action.
\end{abstract}

Keywords Hypergroups · Association schemes · Actions · Maximal actions, $*$-actions $\cdot w$-maximal actions

\section{Introduction}

In this paper we study the maximal actions and $w$-maximal actions of finite hypergroups. Due to the strong similarities between the algebraic structures of finite hypergroups and Bose-Mesner algebras of association schemes, we hope that the study of finite hypergroups will bring a different point of view for the study of association schemes. Actions of finite hypergroups on a finite set, introduced by Sunder and Wildberger [8], provide a way to establish direct connections between finite hypergroups and association schemes. Sunder and Wildberger [8] proved that a finite hypergroup $K$ arises from an association scheme if $K$ admits an irreducible action which is both a maximal action and a *-action. It is well-known that the study of C-algebras and

B. Xu (凶)

Department of Mathematics and Statistics, Eastern Kentucky University, Richmond, KY 40475,

USA

e-mail: bangteng.xu@eku.edu 
table algebras, whose algebraic structures are very similar to the algebraic structure of finite hypergroups, has many interesting applications to association schemes; for example, see [1, 4, 7], and [10].

Hypergroups have been studied by many researchers in various fields for a long time; for references, see [6] or [9]. An action $\pi$ of a finite hypergroup $K$ on a finite set $X$ assigns to every $c_{i} \in K$ an affine map $\pi\left(c_{i}\right)$, which is identified with a column stochastic matrix whose rows and columns are indexed by elements in $X$, such that the product $\pi\left(c_{i}\right) \pi\left(c_{j}\right)$ is a linear combination of $\left\{\pi\left(c_{i}\right) \mid c_{i} \in K\right\}$ with coefficients equal to the structure constants of $c_{i} c_{j}$. Sunder and Wildberger [8] introduced the notion of irreducible actions, maximal irreducible actions, and *-actions. One of their main results in [8] states that if a finite hypergroup $K$ admits an irreducible action which is both a maximal action and a $*$-action, then $K$ arises from an association scheme. In this paper we will first show that an irreducible maximal action must be a *-action, and hence improve Sunder and Wildberger's result [8, Theorem 2.9]. Then we introduce the concept of $w$-maximal actions, a broad class of actions that includes maximal actions and left regular actions. For a $w$-maximal action $\pi: K \rightarrow \operatorname{Aff}(X)$, we will prove that $\pi$ is faithful and $|X| \geq|K|$, and $|K|$ is the best possible lower bound of $|X|$. We will also discuss the strong connectivity of the digraphs induced by a $w$-maximal action, and obtain another point of view to a well-known fact in the theory of association schemes.

The rest of this introductory section gives notation, definitions, theorems, and examples. Throughout this paper, $\mathbb{C}$ denotes the complex numbers, $\mathbb{R}^{+}$the positive real numbers, and $\mathbb{N}$ the positive integers.

Definition 1.1 A finite hypergroup is a distinguished linear basis $K=\left\{c_{0}, c_{1}, \ldots, c_{n}\right\}$ of a complex unital associative *-algebra $\mathbb{C} K$ that satisfies the following conditions, for $0 \leq i, j \leq n$ :

(i) $c_{0}$ is the multiplicative identity of $\mathbb{C} K$;

(ii) $c_{i} c_{j}=\sum_{k=0}^{n} n_{i j}^{k} c_{k}$, where $n_{i j}^{k} \in \mathbb{R}^{+} \cup\{0\}$ for all $k$, and $\sum_{k=0}^{n} n_{i j}^{k}=1$;

(iii) $K$ is a self-adjoint set, i.e., there exists an involutive mapping $i \mapsto i^{*}$ of $\{0,1, \ldots, n\}$ such that $c_{i^{*}}=c_{i}^{*}$; and

(iv) $n_{i j}^{0}>0$ if and only if $j=i^{*}$, where $n_{i j}^{0}$ is the same as in (ii).

Let $K=\left\{c_{0}, c_{1}, \ldots, c_{n}\right\}$ be a finite hypergroup, with $c_{i} c_{j}=\sum_{k=0}^{n} n_{i j}^{k} c_{k}, 0 \leq$ $i, j \leq n$. Then $\left(n_{i^{*} i}^{0}\right)^{-1}$ is called the weight of $c_{i}$, and denoted by $w\left(c_{i}\right)$. Note that for any $i, w\left(c_{i}\right)=w\left(c_{i}^{*}\right)$ [8, Lemma 1.3]. The weight of $K$ is defined by $w(K):=\sum_{i=0}^{n} w\left(c_{i}\right)$.

There are many important examples of finite hypergroups, including the following one.

Example 1.2 Let $X$ be a finite set, and $\left(X,\left\{R_{i}\right\}_{0 \leq i \leq n}\right)$ be an association scheme over $X$ (not necessarily commutative). Let $A_{i}$ be the adjacency matrix with respect to $R_{i}$, and $n_{i}$ be the valency of $R_{i}$. Then $K:=\left\{n_{i}^{-1} A_{i} \mid 0 \leq i \leq n\right\}$ is a finite hypergroup (see [4, Section 2.2]). Note that the weight of $n_{i}^{-1} A_{i}$ is $n_{i}$, and the weight of $K$ is $|X|$. 
The above example is the motivation of the next definition.

Definition 1.3 Let $K=\left\{c_{0}, c_{1}, \ldots, c_{n}\right\}$ be a finite hypergroup with $c_{i} c_{j}=$ $\sum_{k=0}^{n} n_{i j}^{k} c_{k}, 0 \leq i, j \leq n$. If there is an association scheme $\left(X,\left\{R_{i}\right\}_{0 \leq i \leq n}\right)$ over a finite set $X$ with adjacency matrices $A_{i}$ and valencies $n_{i}, 0 \leq i \leq n$, such that

$$
\left(n_{i}^{-1} A_{i}\right) \cdot\left(n_{j}^{-1} A_{j}\right)=\sum_{k=0}^{n} n_{i j}^{k}\left(n_{k}^{-1} A_{k}\right), \quad 0 \leq i, j \leq n,
$$

then we say that the finite hypergroup $K$ arises from the association scheme $\left(X,\left\{R_{i}\right\}_{0 \leq i \leq n}\right)$.

If a finite hypergroup $K=\left\{c_{0}, c_{1}, \ldots, c_{n}\right\}$ arises from an association scheme ( $\left.X,\left\{R_{i}\right\}_{0 \leq i \leq n}\right)$, using the same notation as in Definition 1.3, we have that $w\left(c_{i}\right)=n_{i}$, for all $i$, and $w(K)=|X|$.

Let $X=\left\{x_{1}, x_{2}, \ldots, x_{k}\right\}$ be a finite set, and $V$ be the vector space over $\mathbb{R}$ with basis $X$. The simplex of $X$ is the subset

$s X:=\left\{a_{1} x_{1}+a_{2} x_{2}+\cdots+a_{k} x_{k} \in V \mid a_{i} \geq 0\right.$, for all $i$, and $\left.a_{1}+a_{2}+\cdots+a_{k}=1\right\}$.

An affine map (or a convex map) of $s X$ is a map $T: s X \rightarrow s X$ that satisfies

$$
T(\lambda \alpha+(1-\lambda) \beta)=\lambda T(\alpha)+(1-\lambda) T(\beta), \quad \text { for all } \alpha, \beta \in s X, \lambda \in[0,1] .
$$

The set of all affine maps of $s X$ is denoted by $\operatorname{Aff}(X)$. Let $T \in \operatorname{Aff}(X)$. Then for any $\alpha_{1}, \alpha_{2}, \ldots, \alpha_{t} \in s X$ and any $\lambda_{1}, \lambda_{2}, \ldots, \lambda_{t} \in[0,1]$ with $\lambda_{1}+\lambda_{2}+\cdots+\lambda_{t}=1$, we have that

$$
T\left(\lambda_{1} \alpha_{1}+\lambda_{2} \alpha_{2}+\cdots+\lambda_{t} \alpha_{t}\right)=\lambda_{1} T\left(\alpha_{1}\right)+\lambda_{2} T\left(\alpha_{2}\right)+\cdots+\lambda_{t} T\left(\alpha_{t}\right) .
$$

For any $T \in \operatorname{Aff}(X)$, let $T\left(x_{i}\right)=\sum_{j=1}^{k} \lambda_{j i} x_{j} \in s X$, for all $i$, and let $\operatorname{Mat}(T)$ be the $k \times k$ matrix whose rows and columns are indexed by $x_{1}, x_{2}, \ldots, x_{k}$ and whose $\left(x_{i}, x_{j}\right)$-entry is $\lambda_{i j}$. Then $\operatorname{Mat}(T)$ is a column-stochastic matrix (i.e., the sum of every column is 1 ), and $\mathrm{T}$ is uniquely determined by $\operatorname{Mat}(T)$. Furthermore, $T \rightarrow$ $\operatorname{Mat}(T)$ is a bijection between $\operatorname{Aff}(X)$ and the set of column-stochastic $k \times k$ matrices. For the rest of the paper, we will always identify $\operatorname{Aff}(X)$ with the set of columnstochastic $k \times k$ matrices whose rows and columns are indexed by the elements of $X$. Furthermore, for any matrix $A \in \operatorname{Aff}(X)$ and any $x, y \in X$, the $(x, y)$-entry of A is denoted by $A_{x, y}$.

Definition 1.4 ([8, Definition 2.1]) Let $K=\left\{c_{0}, c_{1}, \ldots, c_{n}\right\}$ be a finite hypergroup with $c_{i} c_{j}=\sum_{k=0}^{n} n_{i j}^{k} c_{k}, 0 \leq i, j \leq n$. An action of $K$ on a finite set $X$ is a mapping

$$
\pi: K \longrightarrow \operatorname{Aff}(X), \quad c_{i} \mapsto \pi\left(c_{i}\right)
$$

such that

(i) $\pi\left(c_{0}\right)=I$, the identity matrix, and

(ii) $\pi\left(c_{i}\right) \pi\left(c_{j}\right)=\sum_{k=0}^{n} n_{i j}^{k} \pi\left(c_{k}\right), 0 \leq i, j \leq n$. 
Sunder and Wildberger [8] introduced the concepts of $*$-actions, irreducible actions, and maximal irreducible actions. Let $\pi: K \rightarrow \operatorname{Aff}(X)$ be an action of a finite hypergroup $K$ on a finite set $X$. Then $\pi$ is called a $*_{\text {-action }}$ if for any $c_{i} \in K$, $\pi\left(c_{i}^{*}\right)=\pi\left(c_{i}\right)^{*}$, the Hermitian adjoint matrix of $\pi\left(c_{i}\right)$. (But here since every entry of $\pi\left(c_{i}\right)$ is a real number, $\pi\left(c_{i}\right)^{*}$ is the transpose matrix of $\pi\left(c_{i}\right)$.) If for any $x, y \in X$, there is $c_{i} \in K$ such that the $(x, y)$-entry of $\pi\left(c_{i}\right), \pi\left(c_{i}\right)_{x, y}$, is not zero, then $\pi$ is called an irreducible action. For an irreducible action $\pi: K \rightarrow \operatorname{Aff}(X)$, by $[8$, Theorem 2.6(i)] we see that

$$
|X| \leq w(K)
$$

Definition 1.5 ([8, Definition 2.8]) An irreducible action $\pi: K \rightarrow \operatorname{Aff}(X)$ of a finite hypergroup $K$ on a finite set $X$ is called a maximal action if $|X|=w(K)$.

Example 1.6 If a finite hypergroup $K=\left\{c_{0}, c_{1}, \ldots, c_{n}\right\}$ arises from an association scheme $\left(X,\left\{R_{i}\right\}_{0 \leq i \leq n}\right)$, using the same notation as in Definition 1.3, then it is clear that

$$
\pi: K \rightarrow \operatorname{Aff}(X), \quad c_{i} \mapsto n_{i}^{-1} A_{i}
$$

is an irreducible action which is both a $*$-action and a maximal action.

One of the main results in [8] states that if a finite hypergroup $K$ admits an irreducible action which is both a maximal action and a *action, then $K$ arises from an association scheme. Our first main result shows that an irreducible maximal action must be a *-action.

Theorem 1.7 Let $K$ be a finite hypergroup and $\pi: K \rightarrow \operatorname{Aff}(X)$ be an irreducible action. If $\pi$ is a maximal action, then $\pi$ is $a *_{\text {-action. }}$

From Theorem 1.7, Theorem 2.9 in [8] can be improved as follows.

Theorem 1.8 A finite hypergroup $K$ arises from an association scheme if and only if $K$ admits an irreducible maximal action.

Theorems 1.7 and 1.8 will be proved in Section 2 .

Now let us introduce the concept of a $w$-maximal action. Let $K=\left\{c_{0}, c_{1}, \ldots, c_{n}\right\}$ be a finite hypergroup. Then

$$
e_{0}:=w(K)^{-1} \sum_{i=0}^{n} w\left(c_{i}\right) c_{i} \in \mathbb{C} K
$$

is the Haar measure of $K$, and $([8,(1.5)])$

$$
e_{0}=e_{0}^{*}=e_{0}^{2}=c_{i} e_{0}=e_{0} c_{i}, \quad \text { for all } i .
$$

Let $\pi: K \rightarrow \operatorname{Aff}(X)$ be an action. Then $\pi$ can be linearly extended to a map from the convex hull of $K$ in $\mathbb{C} K, \operatorname{co}(K)$, to $\operatorname{Aff}(X)$. (Note that $c o(K)$ can be identified in 
a natural manner with the simplex $s K$.) Thus,

$$
\pi\left(e_{0}\right)=w(K)^{-1} \sum_{i=0}^{n} w\left(c_{i}\right) \pi\left(c_{i}\right),
$$

and $\pi\left(e_{0}\right)$ is an idempotent matrix. Furthermore, by [8, Proposition 2.3], $\pi$ is irreducible if and only if for any $x, y \in X, \pi\left(e_{0}\right)_{x, y}=\pi_{x}$, where every $\pi_{x} \in \mathbb{R}^{+}$, and $\sum_{x \in X} \pi_{x}=1$.

Let $\pi: K \rightarrow \operatorname{Aff}(X)$ be an irreducible action, with $\pi\left(e_{0}\right)_{x, y}=\pi_{x}$, for all $x, y \in X$, where $e_{0}$ is the Haar measure of $K$. Then the weight of $x \in X$ with respect to the action $\pi$ is defined by

$$
w_{\pi}(x)=\frac{\pi_{x}}{\min _{y \in X} \pi_{y}} .
$$

So $w_{\pi}(x) \geq 1$ for all $x \in X$. The weight of the set $X$ with respect to the action $\pi$ is defined by

$$
w_{\pi}(X)=\sum_{x \in X} w_{\pi}(x)=\frac{1}{\min _{y \in X} \pi_{y}} .
$$

By [8, Theorem 2.6(i)], we have that

$$
|X| \leq w_{\pi}(X) \leq w(K)
$$

This is the justification of the next definition.

Definition 1.9 An irreducible action $\pi: K \rightarrow \operatorname{Aff}(X)$ of a finite hypergroup $K$ on a finite set $X$ is called a $w$-maximal action if $w_{\pi}(X)=w(K)$.

Clearly any maximal action is a $w$-maximal action. But a $w$-maximal action need not to be a maximal action (see Example 3.1 in Section 3). Example 3.1 also reveals that every finite hypergroup has a $w$-maximal action. Note that if $\pi: K \rightarrow \operatorname{Aff}(X)$ is a $*$-action for a finite hypergroup $K$, then $\pi$ is maximal if and only if $\pi$ is $w$-maximal (Lemma 3.2 in Section 3).

Let $\pi: K \rightarrow \operatorname{Aff}(X)$ be an action, and let $\operatorname{Mat}_{X}(\mathbb{C})$ be the set of all square matrices over $\mathbb{C}$ whose rows and columns are indexed by the elements of $X$. Then $\pi$ can be linearly extended to an algebra homomorphism (still denoted by $\pi$ ) from $\mathbb{C} K$ to $\operatorname{Mat}_{X}(\mathbb{C})$. The algebra homomorphism $\pi: \mathbb{C} K \rightarrow \operatorname{Mat}_{X}(\mathbb{C})$ is a $*$-homomorphism if $\pi$ is a $*$-action.

Definition 1.10 An action $\pi: K \rightarrow \operatorname{Aff}(X)$ is called faithful if the algebra homomorphism $\pi: \mathbb{C} K \rightarrow \operatorname{Mat}_{X}(\mathbb{C})$ is injective.

So an action $\pi: K \rightarrow \operatorname{Aff}(X)$ is faithful if and only if $\left\{\pi\left(c_{i}\right) \mid c_{i} \in K\right\}$ is a linearly independent subset in $\operatorname{Mat}_{X}(\mathbb{C})$. Our next main result is the following

Theorem 1.11 Let $K$ be a finite hypergroup and $\pi: K \rightarrow \operatorname{Aff}(X)$ be a $w$-maximal action. Then $\pi$ is faithful and $|X| \geq|K|$. 
Example 3.1 in Section 3 indicates that $|K|$ is the best possible lower bound of $|X|$ for a $w$-maximal action $\pi: K \rightarrow \operatorname{Aff}(X)$. Also note that a faithful irreducible action need not to be a $w$-maximal action (see Example 3.6 in Section 3).

We will prove Theorem 1.11 in Section 3. We will also introduce the digraphs induced by an action of a finite hypergroup, which are similar to the digraphs induced by an association scheme, and discuss the strong connectivity of these digraphs in Section 3. In particular, the study of strong connectivity of the digraphs induced by a $w$-maximal action yields another point of view to a well-known fact in the theory of association schemes.

\section{Maximal actions}

In this section we will prove Theorems 1.7 and 1.8. Let us show two lemmas first.

Lemma 2.1 Let $K=\left\{c_{0}, c_{1}, \ldots, c_{n}\right\}$ be a finite hypergroup, and let $\pi: K \rightarrow \operatorname{Aff}(X)$ be an irreducible action. Then the following are equivalent.

(i) $\pi$ is a maximal action.

(ii) $\pi\left(e_{0}\right)=w(K)^{-1} J_{|X|}$, where $e_{0}$ is the Haar measure of $K$, and $J_{|X|}$ is the $|X| \times$ $|X|$ matrix whose entries are all 1.

(iii) For any $x \in X, \pi\left(c_{i}\right)_{x, x}=0$, for all $i \neq 0$.

Proof (i) $\Rightarrow$ (ii) Since $\pi$ is irreducible, by [8, Proposition 2.3], for all $x, y \in X$, $\pi\left(e_{0}\right)_{x, y}=\pi_{x}$, where $\pi_{x}>0$, and $\sum_{x \in X} \pi_{x}=1$. Note that for any $x \in X, w_{\pi}(x)=$ $\pi_{x} / \min _{y \in X} \pi_{y} \geq 1$, and $w(K)=|X| \leq \sum_{x \in X} w_{\pi}(x)=w_{\pi}(X) \leq w(K)$ by [8, Theorem 2.6(i)]. So we get that $w_{\pi}(x)=1$ for all $x \in X$. Hence all $\pi_{x}, x \in X$, are equal. Thus $\pi_{x}=|X|^{-1}$, for all $x \in X$, and $\pi\left(e_{0}\right)=|X|^{-1} J_{|X|}$. But $|X|=w(K)$, So (ii) holds.

(ii) $\Rightarrow$ (iii) For any $x \in X, w(K)^{-1}=\pi\left(e_{0}\right)_{x, x}$ and (1.3) imply that

$$
\begin{aligned}
w(K)^{-1} & =w(K)^{-1} \sum_{i=0}^{n} w\left(c_{i}\right) \pi\left(c_{i}\right)_{x, x} \\
& =w(K)^{-1} w\left(c_{0}\right) \pi\left(c_{0}\right)_{x, x}+w(K)^{-1} \sum_{i=1}^{n} w\left(c_{i}\right) \pi\left(c_{i}\right)_{x, x} \\
& =w(K)^{-1}+w(K)^{-1} \sum_{i=1}^{n} w\left(c_{i}\right) \pi\left(c_{i}\right)_{x, x} .
\end{aligned}
$$

Since every $w\left(c_{i}\right)>0$ and every $\pi\left(c_{i}\right)_{x, x} \geq 0$, we have that

$$
\pi\left(c_{i}\right)_{x, x}=0, \quad \text { for all } x \in X, 1 \leq i \leq n
$$

So (iii) holds.

Springer 
(iii) $\Rightarrow$ (i) Since for any $x \in X$ and any $i \neq 0, \pi\left(c_{i}\right)_{x, x}=0$, we see that $\pi\left(e_{0}\right)_{x, x}=w(K)^{-1} \sum_{i=0}^{n} w\left(c_{i}\right) \pi\left(c_{i}\right)_{x, x}=w(K)^{-1}, \quad$ for all $x \in X$.

Hence, [8, Proposition 2.3] implies that $\pi\left(e_{0}\right)_{x, y}=\pi\left(e_{0}\right)_{x, x}=w(K)^{-1}$, for all $x, y \in X$. Thus, $\pi\left(e_{0}\right)=w(K)^{-1} J_{|X|}$. But $\pi\left(e_{0}\right)$ is an idempotent matrix of size $|X|$, we must have that $w(K)=|X|$. Thus, $\pi$ is a maximal action, and (i) holds.

Let $\pi: K \rightarrow \operatorname{Aff}(X)$ be a maximal action. Then Lemma 2.1 implies that

$$
\pi_{x}=\pi\left(e_{0}\right)_{x, y}=|X|^{-1}=w(K)^{-1}, \quad \text { for all } x, y \in X .
$$

Lemma 2.2 Let $K=\left\{c_{0}, c_{1}, \ldots, c_{n}\right\}$ be a finite hypergroup, and let $\pi: K \rightarrow \operatorname{Aff}(X)$ be an action. Then the following hold.

(i) For any $x \in X$,

$$
\left[\pi\left(c_{i}\right) \pi\left(c_{i}^{*}\right)\right]_{x, x} \geq w\left(c_{i}\right)^{-1}, \quad 0 \leq i \leq n .
$$

(ii) If $\pi$ is an irreducible action, with $\pi\left(e_{0}\right)_{x, y}=\pi_{x}$, for all $x, y \in X$, where $e_{0}$ is the Haar measure of $K$, then

$$
0 \leq \pi\left(c_{i}\right)_{x, y} \leq \frac{w(K) \pi_{x}}{w\left(c_{i}\right)}, \quad \text { for all } x, y \in X, 0 \leq i \leq n
$$

Proof (i) Assume that $c_{i} c_{j}=\sum_{k=0}^{n} n_{i j}^{k} c_{k}, 0 \leq i, j \leq n$. Then for any $c_{i}, \pi\left(c_{i}\right) \pi\left(c_{i}^{*}\right)$ $=\sum_{k=0}^{n} n_{i i^{*}}^{k} \pi\left(c_{k}\right)$. Hence,

$$
\left[\pi\left(c_{i}\right) \pi\left(c_{i}^{*}\right)\right]_{x, x}=\sum_{k=0}^{n} n_{i i^{*}}^{k} \pi\left(c_{k}\right)_{x, x} \geq n_{i i^{*}}^{0} \pi\left(c_{0}\right)_{x, x}=w\left(c_{i}^{*}\right)^{-1} .
$$

Since $w\left(c_{i}\right)^{-1}=w\left(c_{i}^{*}\right)^{-1}$ by [8, Lemma 1.3], (2.2) holds.

(ii) For any $i$ and any $x, y \in X, \pi_{x}=\pi\left(e_{0}\right)_{x, y}$ yields that

$$
\pi_{x}=w(K)^{-1} \sum_{j=0}^{n} w\left(c_{j}\right) \pi\left(c_{j}\right)_{x, y} \geq w(K)^{-1} w\left(c_{i}\right) \pi\left(c_{i}\right)_{x, y}, \quad \text { for all } i .
$$

Hence, $0 \leq \pi\left(c_{i}\right)_{x, y} \leq w(K) \pi_{x} w\left(c_{i}\right)^{-1}$, and (2.3) holds.

Now we are ready to prove Theorem 1.7.

Proof of Theorem 1.7 Assume that $K=\left\{c_{0}, c_{1}, \ldots, c_{n}\right\}$, with $c_{i} c_{j}=\sum_{k=0}^{n} n_{i j}^{k} c_{k}$, $0 \leq i, j \leq n$. Let $\pi\left(e_{0}\right)_{x, y}=\pi_{x}$, for all $x, y \in X$. Note that for any $x \in X, w(K) \pi_{x}=$ 1 by (2.1). So by Lemma 2.2(ii),

$$
0 \leq \pi\left(c_{i}\right)_{x, y} \leq w\left(c_{i}\right)^{-1}, \quad \text { for all } x, y \in X, 0 \leq i \leq n .
$$


Now we claim that

$$
\text { for any } x, y \in X, \quad \pi\left(c_{i}^{*}\right)_{y, x} \neq 0 \Rightarrow \pi\left(c_{i}\right)_{x, y} \neq 0, \quad 0 \leq i \leq n \text {. }
$$

If (2.5) is not true, then there are $c_{i}$ and $x, y \in X$ such that $\pi\left(c_{i}^{*}\right)_{y, x} \neq 0$ but $\pi\left(c_{i}\right)_{x, y}=0$. Recall that the sum of every column of $\pi\left(c_{i}^{*}\right)$ is 1 . So $\sum_{z \in X} \pi\left(c_{i}^{*}\right)_{z, x}$ $=1$. Hence $(2.4)$ forces that

$$
\begin{aligned}
{\left[\pi\left(c_{i}\right) \pi\left(c_{i}^{*}\right)\right]_{x, x} } & =\sum_{z \in X} \pi\left(c_{i}\right)_{x, z} \pi\left(c_{i}^{*}\right)_{z, x}=\sum_{z \in X, z \neq y} \pi\left(c_{i}\right)_{x, z} \pi\left(c_{i}^{*}\right)_{z, x} \\
& \leq w\left(c_{i}\right)^{-1}\left(\sum_{z \in X, z \neq y} \pi\left(c_{i}^{*}\right)_{z, x}\right)<w\left(c_{i}\right)^{-1}\left(\sum_{z \in X} \pi\left(c_{i}^{*}\right)_{z, x}\right) \\
& =w\left(c_{i}\right)^{-1} .
\end{aligned}
$$

That is, $\left[\pi\left(c_{i}\right) \pi\left(c_{i}^{*}\right)\right]_{x, x}<w\left(c_{i}\right)^{-1}$, a contradiction to (2.2). Thus, (2.5) must hold. Note that for any $i,\left(c_{i}^{*}\right)^{*}=c_{i}$. So (2.5) yields that

$$
\text { for any } x, y \in X, \quad \pi\left(c_{i}\right)_{x, y} \neq 0 \Longleftrightarrow \pi\left(c_{i}^{*}\right)_{y, x} \neq 0, \quad \text { for all } i \text {. }
$$

Next we show that

$$
\text { for any } x, y \in X, \quad \pi\left(c_{i}\right)_{x, y} \neq 0 \Rightarrow \pi\left(c_{j}\right)_{x, y}=0, \quad \text { for all } j \neq i \text {. }
$$

If (2.7) is not true, then there are $x, y \in X$ and $c_{i}, c_{j}$ such that $\pi\left(c_{i}\right)_{x, y} \neq 0$, $\pi\left(c_{j}\right)_{x, y} \neq 0$, and $j \neq i$. So by (2.6), $\pi\left(c_{j}^{*}\right)_{y, x} \neq 0$. Hence,

$$
\left[\pi\left(c_{i}\right) \pi\left(c_{j}^{*}\right)\right]_{x, x}=\sum_{z \in X} \pi\left(c_{i}\right)_{x, z} \pi\left(c_{j}^{*}\right)_{z, x} \geq \pi\left(c_{i}\right)_{x, y} \pi\left(c_{j}^{*}\right)_{y, x}>0
$$

But on the other hand, $\pi\left(c_{i}\right) \pi\left(c_{j}^{*}\right)=\sum_{k=0}^{n} n_{i j^{*}}^{k} \pi\left(c_{k}\right), n_{i j^{*}}^{0}=0$, and Lemma 2.1 force that

$$
\left[\pi\left(c_{i}\right) \pi\left(c_{j}^{*}\right)\right]_{x, x}=\sum_{k=0}^{n} n_{i j^{*}}^{k} \pi\left(c_{k}\right)_{x, x}=\sum_{k=1}^{n} n_{i j^{*}}^{k} \pi\left(c_{k}\right)_{x, x}=0,
$$

a contradiction. This proves (2.7).

Let $x, y \in X$. If $\pi\left(c_{i}\right)_{x, y} \neq 0$, then for any $j \neq i, \pi\left(c_{j}\right)_{x, y}=0$ by (2.7). Recall that $w(K)^{-1}=\pi\left(e_{0}\right)_{x, y}$ by Lemma 2.1. Hence,

$$
w(K)^{-1}=w(K)^{-1} \sum_{j=0}^{n} w\left(c_{j}\right) \pi\left(c_{j}\right)_{x, y}=w(K)^{-1} w\left(c_{i}\right) \pi\left(c_{i}\right)_{x, y} .
$$

So $\pi\left(c_{i}\right)_{x, y}=w\left(c_{i}\right)^{-1}$. Thus, for any $x, y \in X$ and any $c_{i}$, we have either $\pi\left(c_{i}\right)_{x, y}=$ 0 or $\pi\left(c_{i}\right)_{x, y}=w\left(c_{i}\right)^{-1}$. Since $w\left(c_{i}^{*}\right)^{-1}=w\left(c_{i}\right)^{-1},(2.6)$ yields that $\pi\left(c_{i}^{*}\right)=\pi\left(c_{i}\right)^{*}$, for all $i$. So $\pi$ is a $*$-action, and the theorem is proved. 
The proof of Theorem 1.8 is very easy now. We include a proof here for the convenience of readers. A similar proof can be found in [8]. From Example 1.2, we only need to prove that if a finite hypergroup $K$ admits an irreducible maximal action $\pi: K \rightarrow \operatorname{Aff}(X)$, then $K$ arises from an association scheme. Using the same notation as in the proof of Theorem 1.7 , let $A_{i}:=w\left(c_{i}\right) \pi\left(c_{i}\right), 0 \leq i \leq n$. Then every $A_{i}$ is a $(0,1)$-matrix, and

(i) $A_{0}=I$, the identity matrix,

(ii) $A_{0}+A_{1}+\cdots+A_{n}=J$, the matrix whose entries are all 1 ,

(iii) $A_{i}^{*}=A_{i^{*}}, 0 \leq i \leq n$, and

(iv) $A_{i} A_{j}=\sum_{k=0}^{n} \frac{w\left(c_{i}\right) w\left(c_{j}\right) n_{i j}^{k}}{w\left(c_{k}\right)} A_{k}, 0 \leq i, j \leq n$.

(Note that since all $A_{i}$ are $(0,1)$-matrices, and $A_{0}, A_{1}, \ldots, A_{n}$ are linearly independent, every $w\left(c_{i}\right) w\left(c_{j}\right) n_{i j}^{k} / w\left(c_{k}\right)$ is a nonnegative integer, $0 \leq i, j, k \leq n$.) For any $i \in\{0,1, \ldots, n\}$, define a relation $R_{i}$ on $X$ by

$$
(x, y) \in R_{i} \Longleftrightarrow\left(A_{i}\right)_{x, y} \neq 0, \quad \text { for all } x, y \in X .
$$

Then $\left(X,\left\{R_{i}\right\}_{0 \leq i \leq n}\right)$ is an association scheme. For any $i, A_{i}$ is the adjacency matrix with respect to $R_{i}$, and $w\left(c_{i}\right)$ is the valency of $R_{i}$. Therefore, $K$ arises from the association scheme $\left(X,\left\{R_{i}\right\}_{0 \leq i \leq n}\right)$. This proves Theorem 1.8.

\section{$3 w$-maximal actions}

In this section we will present a characterization of $w$-maximal actions, and use this characterization to prove Theorem 1.11. Then we study the strong connectivity of the digraphs induced by a $w$-maximal action. The so-called $\pi$-minimal point plays an important role in our discussion.

Let $\pi: K \rightarrow \operatorname{Aff}(X)$ be an irreducible action. Then by (1.4), $|X| \leq w_{\pi}(X) \leq$ $w(K)$. Clearly if $\pi$ is a maximal action, then $\pi$ is also a $w$-maximal action. But the next example shows that a $w$-maximal action need not to be a maximal section. This example also shows that every finite hypergroup has a $w$-maximal action.

Example 3.1 Let $K=\left\{c_{0}, c_{1}, \ldots, c_{n}\right\}$ be a finite hypergroup, with $c_{i} c_{j}=\sum_{k=0}^{n} n_{i j}^{k} c_{k}$, $0 \leq i, j \leq n$. Let $X=K$. Then the left-regular action of $K$ is defined by $\pi$ : $K \rightarrow \operatorname{Aff}(X), \pi\left(c_{i}\right)_{c_{j}, c_{k}}=n_{i k}^{j}$. $\pi$ is indeed an action of $K$ by the associativity of multiplication in $\mathbb{C} K$. Let $e_{0}$ be the Haar measure of $K$. By (1.1) and (1.2), $e_{0} c_{i}=e_{0}=w(K)^{-1} \sum_{j=0}^{n} w\left(c_{j}\right) c_{j}$, for all $i$. Hence,

$$
\pi\left(e_{0}\right)=w(K)^{-1}\left(\begin{array}{cccc}
w\left(c_{0}\right) & w\left(c_{0}\right) & \cdots & w\left(c_{0}\right) \\
w\left(c_{1}\right) & w\left(c_{1}\right) & \cdots & w\left(c_{1}\right) \\
\vdots & \vdots & \ddots & \vdots \\
w\left(c_{n}\right) & w\left(c_{n}\right) & \cdots & w\left(c_{n}\right)
\end{array}\right) .
$$

Since every $w\left(c_{i}\right) \geq 1$ and $w\left(c_{0}\right)=1, \pi$ is an irreducible action by [8, Proposition 2.3], and $w_{\pi}(X)=w(K)$. So $\pi$ is a $w$-maximal action. However, $\pi$ need not to be 
a maximal action. $\pi$ is maximal if and only if every $w\left(c_{i}\right)=1$ if and only if $K$ is a group.

Theorem 1.11 states that for a $w$-maximal action $\pi: K \rightarrow \operatorname{Aff}(X),|X| \geq|K|$. The above example reveals that $|K|$ is the best possible lower bound for $|X|$.

For irreducible *-actions, maximal and $w$-maximal are identical. This is the next lemma.

Lemma 3.2 Let $K$ be a finite hypergroup and $\pi: K \rightarrow \operatorname{Aff}(X)$ be an irreducible

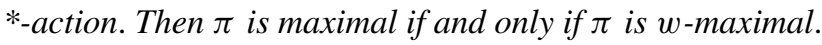

Proof Since $\pi$ is an irreducible $*$-action, $\pi\left(e_{0}\right)=\frac{1}{|X|} J$, where $\mathrm{J}$ is the $|X| \times|X|$ matrix whose entries are all 1. Thus $w_{\pi}(x)=1$, for all $x \in X$. So $w_{\pi}(X)=$ $\sum_{x \in X} w_{\pi}(x)=|X|$. Hence $\pi$ is maximal if and only if it is $w$-maximal.

The next lemma gives a very useful characterization of $w$-maximal actions. This characterization will be needed for the rest of our discussion.

Lemma 3.3 Let $K=\left\{c_{0}, c_{1}, \ldots, c_{n}\right\}$ be a finite hypergroup and $\pi: K \rightarrow \operatorname{Aff}(X)$ be an irreducible action. Then $\pi$ is a $w$-maximal action if and only if there exists $x \in X$ such that $\pi\left(c_{i}\right)_{x, x}=0$, for all $i \neq 0$.

Proof Recall that $\pi\left(e_{0}\right)=w(K)^{-1} \sum_{i=0}^{n} w\left(c_{i}\right) \pi\left(c_{i}\right)$, and for any $x, y \in X, \pi\left(e_{0}\right)_{x, y}$ $=\pi_{x}$, where every $\pi_{x} \in \mathbb{R}^{+}$, and $\sum_{x \in X} \pi_{x}=1$. If $\pi$ is a $w$-maximal action, then $w(K)=w_{\pi}(X)=\frac{1}{\min _{y \in X} \pi_{y}}$. So there is $x \in X$ such that $\pi_{x}=w(K)^{-1}$. But

$$
\begin{aligned}
\pi_{x} & =\pi\left(e_{0}\right)_{x, x}=w(K)^{-1} \sum_{i=0}^{n} w\left(c_{i}\right) \pi\left(c_{i}\right)_{x, x} \\
& =w(K)^{-1}+w(K)^{-1} \sum_{i=1}^{n} w\left(c_{i}\right) \pi\left(c_{i}\right)_{x, x} .
\end{aligned}
$$

Hence, $\pi\left(c_{i}\right)_{x, x}=0$, for all $i \neq 0$.

On the other hand, if there exists $x \in X$ such that $\pi\left(c_{i}\right)_{x, x}=0$, for all $i \neq 0$. Then as above, $\pi_{x}=\pi\left(e_{0}\right)_{x, x}=w(K)^{-1}$. Hence

$$
w_{\pi}(X)=\frac{1}{\min _{y \in X} \pi_{y}} \geq \frac{1}{\pi_{x}}=w(K) .
$$

But by [8, Theorem 2.6(i)], $w_{\pi}(X) \leq w(K)$. Thus, $w_{\pi}(X)=w(K)$, and $\pi$ is $w$ maximal.

Let $K=\left\{c_{0}, c_{1}, \ldots, c_{n}\right\}$ be a finite hypergroup, $\pi: K \rightarrow \operatorname{Aff}(X)$ a $w$-maximal action, and $x \in X$ such that $\pi\left(c_{i}\right)_{x, x}=0$, for all $i \neq 0$. Then $\pi_{x}=w(K)^{-1} \leq \pi_{y}$, for all $y \in X$. Because of the importance of such an $x$, we introduce the following definition. 
Definition 3.4 Let $K=\left\{c_{0}, c_{1}, \ldots, c_{n}\right\}$ be a finite hypergroup and $\pi: K \rightarrow \operatorname{Aff}(X)$ be a $w$-maximal action. Then any $x \in X$ such that $\pi\left(c_{i}\right)_{x, x}=0$, for all $i \neq 0$, is called a $\pi$-minimal point.

Now we are ready to prove Theorem 1.11 .

Proof of Theorem 1.11 Assume that $K=\left\{c_{0}, c_{1}, \ldots, c_{n}\right\}$, with $c_{i} c_{j}=\sum_{k=0}^{n} n_{i j}^{k} c_{k}$, $0 \leq i, j \leq n$. Let $x_{0} \in X$ be a $\pi$-minimal point. Then $\pi\left(c_{i}\right)_{x_{0}, x_{0}}=0$, for all $i \neq 0 \quad$ and $\quad w(K)=w_{\pi}(X)=\left(\pi\left(e_{0}\right)_{x_{0}, y}\right)^{-1}$, for all $y \in X$.

Let us first show that $|X| \geq|K|$. Note that (3.1) yields that

$$
\left[\pi\left(c_{j}^{*}\right) \pi\left(c_{i}\right)\right]_{x_{0}, x_{0}}=\sum_{k=0}^{n} n_{j^{*} i}^{k} \pi\left(c_{k}\right)_{x_{0}, x_{0}}=n_{j^{*} i}^{0}=\delta_{i j} w\left(c_{i}\right)^{-1}, \quad 0 \leq i, j \leq n,
$$

where $\delta_{i j}$ is the Kronecker delta. By (3.2) we can prove the following claims.

Claim 1: For any $i$, there exists at least one $y \in X$ such that $\pi\left(c_{i}\right)_{y, x_{0}} \neq 0$.

If Claim 1 is not true, then there is a $c_{i}$ such that for any $y \in X, \pi\left(c_{i}\right)_{y, x_{0}}=0$. Hence $\left[\pi\left(c_{i}^{*}\right) \pi\left(c_{i}\right)\right]_{x_{0}, x_{0}}=\sum_{y \in X} \pi\left(c_{i}^{*}\right)_{x_{0}, y} \pi\left(c_{i}\right)_{y, x_{0}}=0$, a contradiction to (3.2). So Claim 1 must hold.

Claim 2: For any $i$ and $y \in X$, if $\pi\left(c_{i}\right)_{y, x_{0}} \neq 0$, then $\pi\left(c_{i}^{*}\right)_{x_{0}, y} \neq 0$.

Note that $w(K) \pi_{x_{0}}=1$ by (3.1). Hence, Lemma 2.2(ii) yields that

$$
0 \leq \pi\left(c_{i}\right)_{x_{0}, y} \leq w\left(c_{i}\right)^{-1}, \quad \text { for all } y \in X, 0 \leq i \leq n .
$$

If Claim 2 is not true, then there are $c_{i}$ and $y \in X$ such that $\pi\left(c_{i}\right)_{y, x_{0}} \neq 0$ but $\pi\left(c_{i}^{*}\right)_{x_{0}, y}=0$. Recall that $\sum_{z \in X} \pi\left(c_{i}\right)_{z, x_{0}}=1$. Hence (3.3) forces that

$$
\begin{aligned}
{\left[\pi\left(c_{i}^{*}\right) \pi\left(c_{i}\right)\right]_{x_{0}, x_{0}} } & =\sum_{z \in X} \pi\left(c_{i}^{*}\right)_{x_{0}, z} \pi\left(c_{i}\right)_{z, x_{0}}=\sum_{z \in X, z \neq y} \pi\left(c_{i}^{*}\right)_{x_{0}, z} \pi\left(c_{i}\right)_{z, x_{0}} \\
& \leq w\left(c_{i}^{*}\right)^{-1}\left(\sum_{z \in X, z \neq y} \pi\left(c_{i}\right)_{z, x_{0}}\right)<w\left(c_{i}^{*}\right)^{-1}\left(\sum_{z \in X} \pi\left(c_{i}\right)_{z, x_{0}}\right) \\
& =w\left(c_{i}^{*}\right)^{-1} .
\end{aligned}
$$

That is, $\left[\pi\left(c_{i}^{*}\right) \pi\left(c_{i}\right)\right]_{x_{0}, x_{0}}<w\left(c_{i}^{*}\right)^{-1}=w\left(c_{i}\right)^{-1}$, a contradiction to (3.2). Thus, Claim 2 must hold.

Claim 3: For any $i$ and $y \in X$, if $\pi\left(c_{i}\right)_{y, x_{0}} \neq 0$, then for all $j \neq i, \pi\left(c_{j}\right)_{y, x_{0}}=0$.

If Claim 3 does not hold, then there are $i, j, i \neq j$, and $y \in X$ such that $\pi\left(c_{i}\right)_{y, x_{0}} \neq$ 0 and $\pi\left(c_{j}\right)_{y, x_{0}} \neq 0$. Hence by Claim $2, \pi\left(c_{j}^{*}\right)_{x_{0}, y} \neq 0$. Therefore,

$$
\left[\pi\left(c_{j}^{*}\right) \pi\left(c_{i}\right)\right]_{x_{0}, x_{0}}=\sum_{z \in X} \pi\left(c_{j}^{*}\right)_{x_{0}, z} \pi\left(c_{i}\right)_{z, x_{0}} \geq \pi\left(c_{j}^{*}\right)_{x_{0}, y} \pi\left(c_{i}\right)_{y, x_{0}}>0,
$$

a contradiction to (3.2). So Claim 3 holds. 
Recall that every entry of $\pi\left(e_{0}\right)=w(K)^{-1} \sum_{i=0}^{n} w\left(c_{i}\right) \pi\left(c_{i}\right)$ is positive. So for all $y \in X$, there is $c_{i}$ such that $\pi\left(c_{i}\right)_{y, x_{0}} \neq 0$. Hence, we can define a map

$$
X \longrightarrow K, \quad y \mapsto c_{i} \text { if } \pi\left(c_{i}\right)_{y, x_{0}} \neq 0 .
$$

This map is well-defined by Claim 3, and is surjective by Claim 1 . Therefore, $|X| \geq$ $|K|$.

The faithfulness of $\pi$ also follows from Claims 1 and 3. Here is another direct short proof. If $\pi$ is not faithful, then some $\pi\left(c_{r}\right)$ is a linear combination of $\left\{\pi\left(c_{i}\right) \mid\right.$ $i \neq r\}$. Assume that $\pi\left(c_{r}\right)=\sum_{i \neq r} \alpha_{i} \pi\left(c_{i}\right)$. Then $\pi\left(c_{r}\right) \pi\left(c_{r}^{*}\right)=\sum_{i \neq r} \alpha_{i} \pi\left(c_{i}\right) \pi\left(c_{r}^{*}\right)$. Therefore,

$$
\sum_{k=0}^{n} n_{r r^{*}}^{k} \pi\left(c_{k}\right)=\sum_{i \neq r} \alpha_{i} \sum_{k=0}^{n} n_{i r^{*}}^{k} \pi\left(c_{k}\right)
$$

Note that $n_{i r^{*}}^{0}=0$ for all $i \neq r$. So (3.1) implies that

$$
n_{r r^{*}}^{0}=\sum_{k=0}^{n} n_{r r^{*}}^{k} \pi\left(c_{k}\right)_{x_{0}, x_{0}}=\sum_{i \neq r} \alpha_{i} \sum_{k=0}^{n} n_{i r^{*}}^{k} \pi\left(c_{k}\right)_{x_{0}, x_{0}}=\sum_{i \neq r} \alpha_{i} n_{i r^{*}}^{0}=0,
$$

a contradiction. Hence, $\pi$ must be faithful.

From the proof of Theorem 1.11, we have the following

Corollary 3.5 Let $K=\left\{c_{0}, c_{1}, \ldots, c_{n}\right\}$ be a finite hypergroup, $\pi: K \rightarrow \operatorname{Aff}(X) a$ $w$-maximal action, and $x_{0} \in X$ a $\pi$-minimal point. Then the following hold.

(i) For any $y \in X$, there is a unique $c_{i}$ such that $\pi\left(c_{i}\right)_{y, x_{0}} \neq 0$.

(ii) For any $y \in X$, if $\pi\left(c_{i}\right)_{y, x_{0}} \neq 0$, then $\pi\left(c_{i}^{*}\right)_{x_{0}, y} \neq 0$.

The next example reveals that a faithful action need not to be a $w$-maximal action.

Example 3.6 Let $K=\left\{c_{0}, c_{1}, c_{2}\right\}$ be a finite hypergroup with

$$
c_{1}^{2}=\frac{1}{3} c_{0}+\frac{2}{3} c_{2}, \quad c_{1} c_{2}=c_{2} c_{1}=c_{1}, \quad c_{2}^{2}=\frac{1}{2} c_{0}+\frac{1}{2} c_{2}, \quad \text { and } c_{i}^{*}=c_{i}, \text { for all } i .
$$

Then the action $\pi: K \rightarrow \operatorname{Aff}(X)$, where $X=\left\{x_{1}, x_{2}, x_{3}, x_{4}\right\}$, defined by

$$
\begin{aligned}
& \pi\left(c_{0}\right)=\left(\begin{array}{llll}
1 & 0 & 0 & 0 \\
0 & 1 & 0 & 0 \\
0 & 0 & 1 & 0 \\
0 & 0 & 0 & 1
\end{array}\right), \quad \pi\left(c_{1}\right)=\frac{1}{2}\left(\begin{array}{llll}
0 & 0 & 1 & 1 \\
0 & 0 & 1 & 1 \\
1 & 1 & 0 & 0 \\
1 & 1 & 0 & 0
\end{array}\right), \\
& \pi\left(c_{2}\right)=\frac{1}{4}\left(\begin{array}{llll}
1 & 3 & 0 & 0 \\
3 & 1 & 0 & 0 \\
0 & 0 & 1 & 3 \\
0 & 0 & 3 & 1
\end{array}\right)
\end{aligned}
$$

is a faithful irreducible action. Note that every $\pi\left(c_{i}\right)$ is symmetric. So $\pi$ is a $*$-action. Hence $w_{\pi}(X)=|X|=4<6=w(K)$. Therefore, $\pi$ is not a $w$-maximal action. 
Let $K=\left\{c_{0}, c_{1}, \ldots, c_{n}\right\}$ be a finite hypergroup. For any $a=\sum_{i=0}^{n} \alpha_{i} c_{i} \in \mathbb{C} K$, define $\operatorname{Supp}(a)=\left\{c_{i} \mid \alpha_{i} \neq 0\right\}$. A nonempty subset $S$ of $K$ is a subhypergroup of $K$ if for any $c_{i}, c_{j} \in S, \operatorname{Supp}\left(c_{i} c_{j}\right) \subseteq S$. If $S$ is a subhypergroup of $K$, then $c_{0} \in S$, $S$ is closed under the involution * of $K$, and $S$ itself is a hypergroup. Note that for any $c_{i} \in S$, the weight of $c_{i}$ as an element in $\mathrm{S}$ is the same as the weight of $c_{i}$ as an element in $K$. So $w(S) \leq w(K)$, and $w(S)=w(K)$ if and only if $S=K$.

Let $S$ be a subhypergroup of $K$, and $\pi: K \rightarrow \operatorname{Aff}(X)$ be an action. Then, via restriction, $\pi$ induces an action

$$
\pi_{S}: S \rightarrow \operatorname{Aff}(X), \quad c_{i} \mapsto \pi\left(c_{i}\right), \text { for all } c_{i} \in S,
$$

called the restriction of $\pi$ to $S$.

Proposition 3.7 Let $S$ be a proper subhypergroup of $K$, and $\pi: K \rightarrow \operatorname{Aff}(X)$ be a $w$-maximal action. Then the restriction $\pi_{S}$ is not an irreducible action.

Proof Toward a contradiction, assume that $\pi_{S}$ is an irreducible action. Let $K=$ $\left\{c_{0}, c_{1}, \ldots, c_{n}\right\}$, and $x_{0} \in X$ be a $\pi$-minimal point. Then for any $i \neq 0, \pi\left(c_{i}\right)_{x_{0}, x_{0}}=0$. Hence, for any $c_{i} \in S$ with $i \neq 0, \pi_{S}\left(c_{i}\right)_{x_{0}, x_{0}}=\pi\left(c_{i}\right)_{x_{0}, x_{0}}=0$. So $\pi_{S}$ is also a $w$-maximal action. For any $c_{i} \in K$, there exists $y \in X$ such that $\pi\left(c_{i}\right)_{y, x_{0}} \neq 0$ by Claim 1 in the proof of Theorem 1.11. Since $\pi_{S}$ is irreducible, there is $c_{j} \in S$ such that $\pi_{S}\left(c_{j}\right)_{y, x_{0}} \neq 0$. So $\pi\left(c_{j}\right)_{y, x_{0}} \neq 0$, and we must have that $c_{i}=c_{j} \in S$ by Corollary 3.5(i). Hence, every $c_{i} \in K$ is also in $S$, and $K=S$, a contradiction to the assumption that $S$ is a proper subhypergroup of $K$. Therefore, $\pi_{S}$ is not an irreducible action.

Proposition 3.7 is not necessarily true if the action $\pi$ is not $w$-maximal, as indicated by the next example.

Example 3.8 Let $K$ be the same as in Example 3.6. Then the action $\pi: K \rightarrow \operatorname{Aff}(X)$, where $X=\left\{x_{1}, x_{2}\right\}$, defined by

$$
\pi\left(c_{0}\right)=\left(\begin{array}{ll}
1 & 0 \\
0 & 1
\end{array}\right), \quad \pi\left(c_{1}\right)=\frac{1}{2}\left(\begin{array}{ll}
1 & 1 \\
1 & 1
\end{array}\right), \quad \pi\left(c_{2}\right)=\frac{1}{4}\left(\begin{array}{ll}
1 & 3 \\
3 & 1
\end{array}\right)
$$

is an irreducible action. Note that $w_{\pi}(X)=2<6=w(K)$. So $\pi$ is not a $w$-maximal action. Let $S=\left\{c_{0}, c_{2}\right\}$. Then $S$ is a proper subhypergroup of $K$, and the restriction action

$$
\pi_{S}: S \rightarrow \operatorname{Aff}(X), \quad c_{0} \mapsto \pi\left(c_{0}\right), c_{2} \mapsto \pi\left(c_{2}\right)
$$

is also irreducible. It is also interesting to note that although $S$ is a proper subhypergroup of $K$,

$$
w_{\pi_{S}}(X)=2=w_{\pi}(X) .
$$

Similar to the digraphs induced by association schemes (see [3] or [4]), the digraphs induced by actions of hypergroups can be defined as follows. Let $K=$ 
$\left\{c_{0}, c_{1}, \ldots, c_{n}\right\}$ be a finite hypergroup, and $\pi: K \rightarrow \operatorname{Aff}(X)$ be an action. Then for any $i \neq 0, \pi\left(c_{i}\right)$ induces a digraph $\Gamma_{\pi\left(c_{i}\right)}$ such that

$$
V\left(\Gamma_{\pi\left(c_{i}\right)}\right)=X, \quad E\left(\Gamma_{\pi\left(c_{i}\right)}\right)=\left\{(x, y) \mid \pi\left(c_{i}\right)_{x, y} \neq 0\right\} .
$$

For any $c_{i} \in K$, let $K_{c_{i}}:=\cup_{r \in \mathbb{N}} \operatorname{Supp}\left(c_{i}^{r}\right)$. Then $K_{c_{i}}$ is a subhypergroup of $K$. The next lemma reveals that the strong connectivity of the digraphs $\Gamma_{\pi\left(c_{i}\right)}$ is determined by the restriction action $\pi_{K_{c_{i}}}$.

Lemma 3.9 Let $K=\left\{c_{0}, c_{1}, \ldots, c_{n}\right\}$ be a finite hypergroup, and $\pi: K \rightarrow \operatorname{Aff}(X)$ be an action. Then the digraph $\Gamma_{\pi\left(c_{i}\right)}$ is strongly connected if and only if the restriction $\pi_{K_{c_{i}}}$ is an irreducible action.

Proof First assume that $\Gamma_{\pi\left(c_{i}\right)}$ is strongly connected. Let $x, y \in X$. Then there is a path (of length $r$ ) from $x$ to $y$. Hence $\left[\pi\left(c_{i}\right)^{r}\right]_{x, y} \neq 0$. Suppose that $c_{i}^{r}=\sum_{j=0}^{n} \alpha_{j} c_{j}$. Then

$$
\left[\pi\left(c_{i}\right)^{r}\right]_{x, y}=\pi\left(c_{i}^{r}\right)_{x, y}=\sum_{j=0}^{n} \alpha_{j} \pi\left(c_{j}\right)_{x, y} .
$$

Hence, there is $\alpha_{j} \neq 0$ with $\pi\left(c_{j}\right)_{x, y} \neq 0$. Note that $c_{j} \in K_{c_{i}}$. So $\pi_{K_{c_{i}}}\left(c_{j}\right)_{x, y}=$ $\pi\left(c_{j}\right)_{x, y} \neq 0$. Hence the restriction $\pi_{K_{c_{i}}}$ is irreducible.

On the other hand, assume that $\pi_{K_{c_{i}}}$ is an irreducible action. Let $x, y \in X$. Then there exists $c_{j} \in K_{c_{i}}$ such that $\pi\left(c_{j}\right)_{x, y}=\pi_{K_{c_{j}}}\left(c_{j}\right)_{x, y} \neq 0$. Assume that $c_{j} \in \operatorname{Supp}\left(c_{i}^{r}\right)$ for some $r \in \mathbb{N}$. Then as above, $\left[\pi\left(c_{i}\right)^{r}\right]_{x, y} \neq 0$. So there is a path (of length $r$ ) from $x$ to $y$. Therefore, $\Gamma_{\pi\left(c_{i}\right)}$ is strongly connected.

The next proposition generalizes a well-known fact in the theory of association schemes as well as a similar property for table algebras (see [2, Proposition 4.3]; a table algebra (A, B) has a unique rescaling $\left(A, \mathbf{B}^{\prime}\right)$ such that $\mathbf{B}^{\prime}$ is a finite hypergroup, see [5]). Our short proof here provides another point of view to this result.

Proposition 3.10 Let $K=\left\{c_{0}, c_{1}, \ldots, c_{n}\right\}$ be a finite hypergroup, and $\pi: K \rightarrow$ $\operatorname{Aff}(X)$ a w-maximal action. Then $\Gamma_{\pi\left(c_{i}\right)}$ is strongly connected if and only if $K_{c_{i}}=K$.

Proof By Lemma 3.9, the digraph $\Gamma_{\pi\left(c_{i}\right)}$ is strongly connected if and only if the restriction $\pi_{K_{c_{i}}}$ is an irreducible action. Since $\pi$ is $w$-maximal, by Proposition 3.7, the restriction action $\pi_{K_{c_{i}}}$ is irreducible if and only if $K_{c_{i}}=K$. So $\Gamma_{\pi\left(c_{i}\right)}$ is strongly connected if and only if $K_{c_{i}}=K$.

Proposition 3.10 is not necessarily true if the action $\pi$ is not $w$-maximal. For example, Let $K$ and $\pi: K \rightarrow \operatorname{Aff}(X)$ be the same as in Example 3.8. Then $\pi$ is not a $w$-maximal action. Although $\Gamma_{\pi\left(c_{2}\right)}$ is strongly connected, $K_{c_{2}}=\left\{c_{0}, c_{2}\right\} \neq K$.

\section{References}

1. Arad, Z., Erez, Y., \& Muzychuk, M. (2003). On even generalized table algebras. Journal of Algebraic Combinatorics, 17(2), 163-170. 
2. Arad, Z., Fisman, E., \& Muzychuk, M. (1999). Generalized table algebras. Israel Journal of Mathematics, 114, 29-60.

3. Brouwer, A., Cohen, A., \& Neumaier, A. (1989). Distance-regular graphs. Berlin: Springer.

4. Bannai, E., \& Ito, T. (1984). Algebraic combinatorics I: association schemes. Menlo Park: Benjamin/Cummings.

5. Blau, H., \& Zieschang, P. (2004). Sylow theory for table algebras, fusion algebras, and hypergroups. Journal of Algebra, 273, 551-570.

6. Obata, N., \& Wildberger, N. J. (1996). Generalized hypergroups and orthogonal polynomials. Nagoya Mathematical Journal, 142, 67-93.

7. Suzuki, H. (1998). Imprimitive Q-polynomial association schemes. Journal of Algebraic Combinatorics, 7, 165-180.

8. Sunder, V. S., \& Wildberger, N. J. (2003). Actions of finite hypergroups. Journal of Algebraic Combinatorics, 18(2), 135-151.

9. Wildberger, N. J. (1997). Duality and entropy for finite abelian hypergroups and fusion rule algebras. The Journal of the London Mathematical Society, 56(2), 275-291.

10. Xu, B. (2006). Table algebras with multiple P-polynomial structures. Journal of Algebraic Combinatorics, 23, 377-393. 